\title{
Probing the pH-dependent chain dynamics of poly(acrylate acid) in concentrated solution by using a cationic AIE fluorophore
}

\author{
ZHANG Shuang $^{1}$, YAN JiMing ${ }^{1}$, QIN AnJun ${ }^{1}$, SUN JingZhi ${ }^{1 *}$ \\ \& TANG Ben Zhong ${ }^{1,2 *}$
}

1 MOE Key Laboratory of Macromolecular Synthesis and Functionalization, Department of Polymer Science and Engineering, Zhejiang University, Hangzhou 310027, China

2 Department of Chemistry, The Hong Kong University of Science \& Technology, Clear Water Bay, Hong Kong, China

*Corresponding authors (email: sunjz@zju.edu.cn; tangbenz@ust.hk)

Received May 31, 2013; accepted June 18, 2013; published online July 19, 2013

全文见: Zhang S, Yan JM, Qin AJ, Sun JZ, Tang BZ. Probing the pH-dependent chain dynamics of poly(acrylate acid) in concentrated solution by using a cationic AIE fluorophore. Sci China Chem, 2013, 56(9): 1253-1257

\begin{abstract}
We report a novel strategy to study the chain dynamics of poly(acrylic acid) (PAA) in a relative concentrated solution $(1.0 \mathrm{~g} / \mathrm{L})$. The strategy is based on the fluorescent probe (DCTPE) with unique aggregation-induced emission (AIE) characteristics. Free DCTPE molecules are non-emissive in aqueous solution, but they become highly emissive when trapped in polymer coils. The fluorescence intensity is proportional to the efficiency of trapping DCTPE molecules in polymer coils. By correlation the change of fluorescence intensity with the variation of $\mathrm{pH}$ value (from 1.78 to 12.06), the PAA chain's dynamics in the relatively concentrated solution have been elucidated into three processes. In the $\mathrm{pH}$ range from 12.06 to 6.0, PAA chains take an extended and non-folding conformation. Changing $\mathrm{pH}$ from 6.0 to 3.86 , PAA chains are partially protonated and loosely packed polymer coils are formed. Further lowering the $\mathrm{pH}$ value of the solution (from 3.86 to 1.78), protonated segments dominate the PAA chains, and at the same time, the intermolecular hydrogen bonding takes effect, thus the polymer chains posses in the conformation of more compact coils.
\end{abstract}

Keywords: polyelectrolyte, polymer dynamics, coils, concentrated solution, aggregation-induced emission

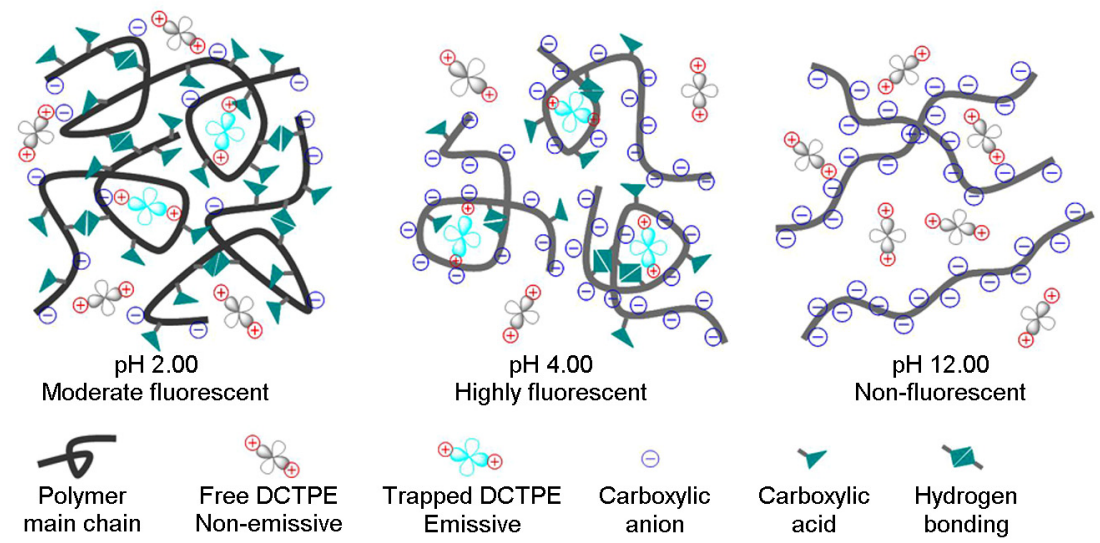

An illustration of the dynamic conformation changes of PAA chains at different $\mathrm{pH}$ values. PAA concentration: $1 \mathrm{~g} / \mathrm{L}$. 